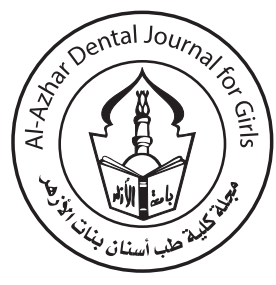

\title{
Evaluation of Fascin Expression in Selected Salivary Gland Tumors (An immunohistochemical study)
}

\author{
Alaa E.Ebrahim ${ }^{(1)}$, Nadia A. Radi ${ }^{(2)}$ and Eman A. Abo Hager ${ }^{(3)}$
}

Codex : $14 / 1901$

azhardentj@azhar.edu.eg

http://adjg.journals.ekb.eg

DOI: $10.21608 /$ adjg.2019.6078.1052

\section{KEYWORDS}

Fascin, immunohistochemistry, salivary gland tumors

\begin{abstract}
Purpose: Fascin taken countless attention between cytoskeletal proteins; this because it is not normally present in epithelial tissues, and it's role in epithelial to mesenchymal transition leads to motility and invasion of cancer cells. The purpose of this work was to estimate the expression of the cytoskeleton-associated protein fascin as a prognostic indicator of invasiveness in salivary gland neoplasms and also, to relate its expression with the biological behavior of the salivary gland neoplasms by mean of the immunohistochemical technique. Material and method: 50 cases of salivary gland tissues were collected as paraffin embedded blocks: 20 benign cases (10 pleomorphic adenoma and 10 Warthin's tumors) and 25 malignant cases (15 cylindroma in addition to 10 mucoepidermoid carcinomas). Additionally, 5 normal salivary gland tissues. An immunohistochemical investigation using fascin antibody was done for all specimens. Results: The mean area percent of immunoexpression was greatest in malignant variants of salivary gland tumors, followed by benign variants of salivary gland tumors and the least value recorded in normal salivary gland tissue. Conclusion: According to the current study, fascin participates in the development of salivary gland tumors and positively related to the most aggressive patterns of malignant neoplasms.
\end{abstract}

Paper extracted From Master thesis entitled "Evaluation of Fascin Expression in selected salivary gland tumors (immunohistochemical study).

\section{INTRODUCTION}

Salivary gland tumors are a set of diseases which represent important lesions of oral and maxillofacial pathology ${ }^{(1)}$.They represent the main histologically heterogeneous group of human tumors with probably the greatest morphological diversity and cellular features and considering a specific tumor into a single category is neither always dependable nor straightforward ${ }^{(2)}$. So any mistakes in the diagnosis are

1. Demonstrator of Oral and Dental Pathology. Faculty of Dental Medicine for Girls. Al Azhar University.

2. Lecturer of Oral and Dental Pathology, Faculty, of Dental Medicine for Girls. Al Azhar University.

3. Professor of Oral and Dental Pathology, Faculty of Dental Medicine for Girls. Al Azhar University. 
equal to the poor prognosis of the tumor considering recurrence and metastasis ${ }^{(3)}$. Parotid tumors include eighty percent to eighty fivepercent of all salivary gland tumors and they are almost benign. However, Submandibular gland tumors represent ten percent of all salivary gland tumors. Fewer than one percent of salivary neoplasms take place in the sublingual glands, nonetheless seventy five percent to eighty percent of these are malignant. Other salivary gland tumors occuring in minor salivary glands especially the palate, and nearly all these were malignant ${ }^{(4)}$. Identifying cellular markers for differentiation, recurrence determination, prognosis and treatment planning of such tumors is valuable.

Fascin is an actin protein of $55 \mathrm{kDa}$, encrypted by a gene located on chromosome 7p22 in humans. It has three forms: Fascin 1, also named fascin expressed by stromal and nervous tissues ${ }^{(5,6)}$. Fascin taken count less attention between cytoskeletal proteins; this because it is not normally present in epithelial tissues, and it's role in epithelial to mesenchymal taransition leads to motility and invasion of cancer cells ${ }^{(7)}$. Also, it plays an important role in the formation of invadopodia, which is extremely energetic actin that is gathered by one as well as the other actin-related protein complex and formins; similarly having constituent of membrane machinery alike cortactin protein allowing invasion of tumor into the substances where the cells embedded and interrupt epithelial connections ${ }^{(8)}$. By way of fascin is known to be exceedingly present in various tumor cell types, this advocates that tumor cells might precisely deplete elevated fascin stages to effective cell movement. This would take action to organize both permanence of cell protuberances for instance filopodia to discover the media, nuclear movement besides distortion to allow invasion ${ }^{(9)}$. The overexpression of fascin was found related with poor prognosis in many malignant tumors for example colon cancer, gastric cancer, oral carcinoma of squamous cell, cancer of the lung, carcinoma of squamous cell of esophageus besides breast cancer ${ }^{(10-14)}$.Therefore, the present study has been done aiming to evaluate the expression of the cytoskeleton-associated protein fascin as a prognostic indicator of invasiveness in salivary gland neoplasms and also, to correlate its expression with the biological behavior of the salivary gland neoplasms via mean of the immunohistochemical technique.

\section{MATERIALS AND METHODS}

\section{Case selection}

The specimens gotten for this study were retrieved as a paraffin embedded blocks from the archives of Oral and Dental Pathology Department, Faculty of Dental Medicine for Girls, Al-Azhar University and Oral Pathology Department, Faculty of Dentistry, Alexandria University.

The cases distributed into three sets: Group I, control group including five cases of normal salivary gland tissue. Group II, benign salivary gland tumors, including 20 cases divided into, 10 cases of pleomorphic adenoma (PA), 10 cases of Warthin's tumor. Group III, malignant salivary gland tumors, including 25 cases divided into, 10 cases of mucoepidermoid carcinoma (MEC) with different grades, and 15 cases of adenoid cystic carcinoma (ACC) representing the different patterns.

\section{Histological analysis}

Using H\&E for reevaluation of the aforementioned cases was carried out to confirm their diagnosis, and establish the histopathologic grading according to $\mathrm{WHO}^{(15)}$.

\section{Immunohistochemical evaluation}

The streptavidin-biotin immunohistochemical method smeared to $4 \mu \mathrm{m}$ sections thickness which fixed on electro positive glass slips ${ }^{(16)}$. They first overnight deparaffinized and cultivated with xylene then, rehydrated gradually with ethanol afterward Phosphate buffered saline (PBS) wash. Masking the peroxidase action was done by $3 \%$ hydrogen peroxide $\left(\mathrm{H}_{2} \mathrm{O}_{2}\right)$ for five minutes at room heat. For antigen recovery, tissue sections were put in a cut-glass jar containing sodium citrate buffer ( $\mathrm{pH}$ 6.0) and heated in a microwave oven two times for five minutes. The slides then permitted to cool and bathed with PBS. 
Staining for fascin antibody was performed consuming fascin mouse monoclonal antibody (Clone D-10: SC-46675, Dako, Denmark). The dilution used was 1:50 in phosphate buffered saline.

Recognition was done by the worldwide set by laundry slips in PBS for five minutes and keep warm with antibody that was biotinylated goat serum conjugated rabbit and mouse sera for thirty minutes. Sections were then clear up to five minutes in PBS then antigen-antibody monitoring by diaminobenzidine in PBS containing fourty percent $\mathrm{H} 2 \mathrm{O} 2$. Sections were eroded under running tap water for ten minutes, then discolored with Mayer's hematoxylin and mounted.

\section{Histomorphometric evaluation}

Immunoreactivity for both fascin-1 was assessed by calculate approximately the ratio of stained cells in relative to the zone inspected in every field, by means of a Leica system for image analysis. The system composed of a color videotape, screen, the disc of computer attached to light microscope and Leica software. The analyzer was rectified mechanically to change the measurement parts (pixels) into micrometer units. The zone besides space fraction of both ezrin in addition fascin reactive areas sedated with reference to a fixed calculating border of area 11434.9 micrometer $^{2}$ using intensification ( $\mathrm{x} 200$ ). Consuming the color detection, positive parts were covered by a blue binary color. Ten fields per each slide measured to be histomorphometrically estimated. Then mean values achieved for all samples.

\section{Statistical Examination}

Information was obtainable as mean and standard deviation (SD) measures. Analysis of variance test used to relate means of more than two groups. Tukey Kramer multiple comparisons were used in the procedure of pairwise comparisons among the collections when Analysis of variance test is of importance. $\mathrm{P}$ value is important if not as much of or identical to $0.05(\mathrm{P} \leq 0.05)$. Arithmetical investigation was done by in-state graph pad version 3.10 and Microsoft@ excel 2007.

\section{RESULTS}

\section{Histopathological findings:}

The normal salivary gland tissue specimens are formed of acini of serous cells with the duct system. Connective tissue septa were seen surrounding the lobules of glandular structures (Fig., 1A). The pleomorphic adenoma cases showed a mixture of glandular epithelium and myoepithelial cells within a mesenchymal-like background. The epithelial element showed solid sheets, strands of epithelial cells and duct-like structures, some cases showed chondroid tissue. Other areas showed a variable number of myoepithelial cells which appeared as stellate-shaped or spindle cells present in mucoid or myxoid tissue (Fig., 1B). Warthin's tumor revealed multiple cystic spaces surrounded by the ductal epithelium of double row of cells showing abundant, finely granular eosinophilic cytoplasm. The inner luminal layer was tall columnar cells with centrally placed palisaded and slightly hyperchromatic nuclei. Beneath this, a layer of cuboidal cells with more vesicular nuclei was seen. The lining epithelium demonstrates multiple papillary infolding that protrudes into cystic spaces. The connective tissue contains lymphoid stroma that frequently showed germinal centers formation (Fig., 1C).

Some cases of low-grade MEC showed large masses of cells that secrete mucous with few epidermal cells, while other cases revealed small adjacent cysts or large cystic spaces which were surrounded by cells that secrete mucous intermingled with few epidermal cells. Little cellular atypia and mitotic figures were evident in epidermoid cells (Fig., 2G). High-grade MEC showed solid islands of epidermoid and intermediate cells demonstrated considerable cellular and nuclear pleomorphism, nuclear hyperchromatism and mitotic figures. The sheets and cords of cells were embedded in scanty connective tissue stroma (Fig., 2H).

The cribriform pattern of ACC revealed cystic spaces surrounded by small basaloid cells and showed slightly basophilic mucinous areas. The cells revealed scanty cytoplasm and darkly stained 
hyperchromatic nuclei (Fig., 3K). While the tubular pattern showed ductal and myoepithelial cells arranged in rows or small duct-like structures, these duct cells were present in hyaline connective tissue (Fig., 3L). The solid pattern showed island or sheets of small uniform and undifferentiated tumor cells. The tumor cells were embedded in little eosinophilic hyalinized stroma, very few spaces or glandular structure were present (Fig., 3M).

\section{Immunohistochemical findings:}

Fascin immunostaining was noticed in the nucleus and cytoplasm of ductal cells plus the surrounding myoepithelial cells in the normal salivary gland. (Fig., 1D). Regarding benign neoplasm, fascin immunostaining in PA was seen in the cytoplasm and nucleus of epithelial tissue elements, duct-like structures and also seen in chondroid tissue, myoepithelial cells and myxoid tissue (Fig., 1E). In Warthin's tumor fascin was seen in the cytoplasm of the cells of ductal epithelium, while it was present in nucleus and cytoplasm of basal cells, also in the lymphocyte of germinal centers (Fig., 1F). Regarding malignant neoplasm, In MEC, for both low and high grades, fascin immunostaining was seen in cytoplasm and nucleus of epidermoid cells and stromal fibroblasts, while the mucous cells are negative (Fig.,2 I, J). In ACC fascin immunostaining appear in the cytoplasm of the neoplastic cells in cribriform and tubular patterns and nuclear and cytoplasmic staining in solid pattern (Fig.,3 N, O, P).

\section{Statistical results:}

The highest mean area percent of immuneexpression was recorded in high-grade mucoepidermoid carcinoma, followed by the solid pattern of cylindroma. The lowermost mean value logged in normal salivary tissue. Analysis of variance examination shown that the dissimilarity among all groups stayed extremely statistically significant $(\mathrm{p}<0.0001)$. Tukey's post hoc experiment showed there is no significant difference between the solid pattern of cylindroma and high and low-grade MEC. Moreover, high-grade MEC was not significantly different from tubular and cribriform cylindroma.
In addition, cribriform cylindroma was not significantly different from pleomorphic adenoma and papillary cyst adenoma lymphomatosum. Warthin's tumor was not significantly different from normal salivary tissue (Table 1, Fig., 4)

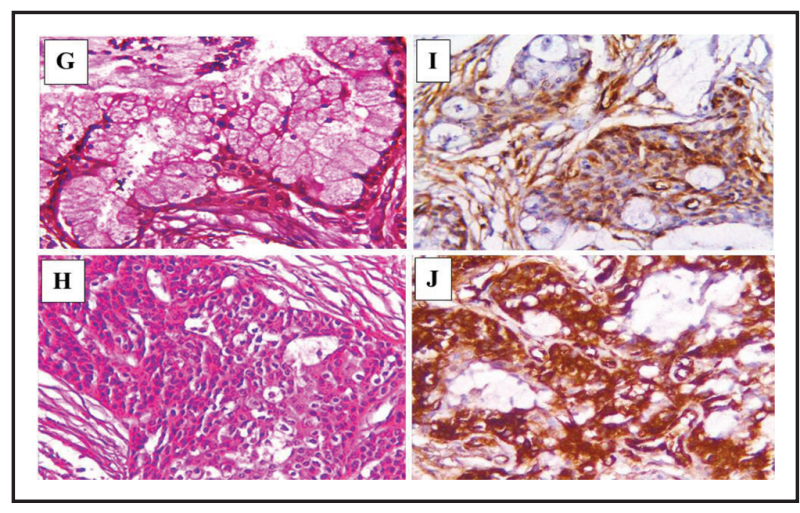

Fig. (1) Normal salivary gland tissue, A, pleomorphic adenoma showing duct like structure, myxoid and chondroid tissue, B, Warthin's tumor showing cystic spaces lined by double row of salivary epithelium with lymphoid stroma, C, (A-C, H\&E X200). Fascin immunostaining detected in the nucleus and cytoplasm of ductal cells in normal salivary gland, D, Fascin immunostaning of cytoplasm of epithelial cells, of myoepithelial cells and chonroid tissue cells, E, Fascin immunostaining of cytoplasm of double row of ductal epithelial cells, F, (D-F, Fascin, X200).

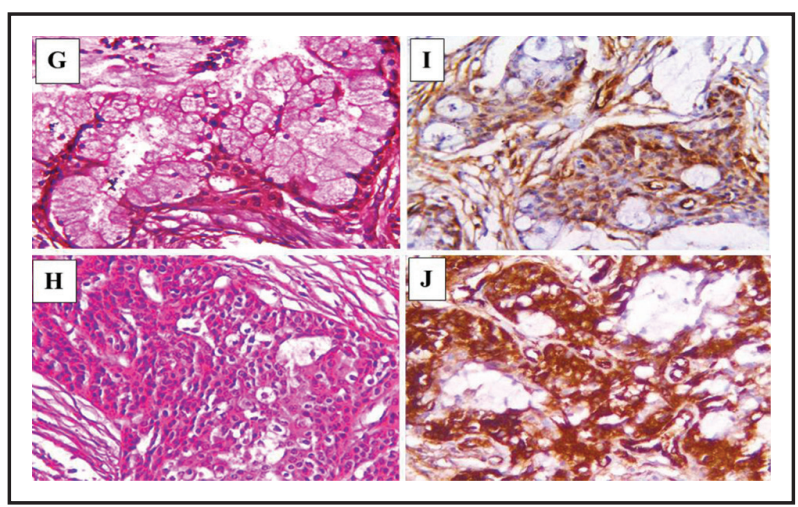

Fig. (2) Low grade MEC showing nests of mucous secreting cells and few epidermoid cells, G. High grade MEC showing numerous epidermoid cells and few mucous secreting cells, H. (G-H, H\&E X 200). Fascin immunostaining of cytoplasm of epidermoid cells and negative mucous secreting cells in low grade MEC, I. nuclear and cytoplasmic immunostaining of epidermoid cells in high grade MEC, J, (I-J, Fascin, X200). 


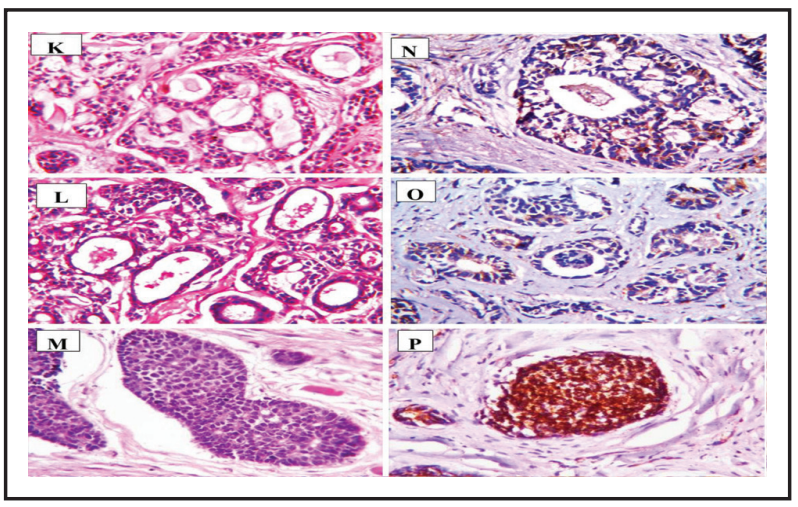

Fig. (3) Adenoid cystic carcinoma showing hyperchromatic cells forming cribriform structure, $\mathrm{K}$, tubular structure, L, solid structure, M, (K-M, H\&E X 200). Fascin immunostaining of cytoplasm of neoplastic cells of cribriform structure, $\mathrm{N}$, tubular structure, $\mathrm{O}$, Fascin immunostaining of nucleus and cytoplasm of neoplastic cells of solid structure, P, (N-P, Fascin, X200).

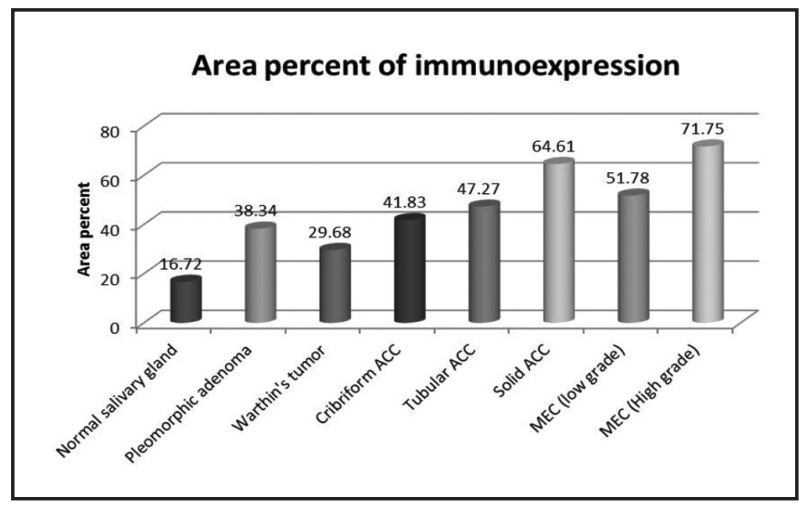

Fig. (4) Column chart showing mean area percent of immunoexpression of different groups

Table (1): Area percent of immunoexpression in different groups (ANOVA test)

\begin{tabular}{|c|c|c|c|c|c|c|c|}
\hline & \multirow{2}{*}{ Mean } & \multirow{2}{*}{$\begin{array}{l}\text { Standerd. } \\
\text { Deviation }\end{array}$} & \multirow{2}{*}{$\begin{array}{c}\text { Standerd. } \\
\text { Error }\end{array}$} & \multicolumn{2}{|c|}{ 5\% CIfor Mean } & \multirow{2}{*}{ Minimum } & \multirow{2}{*}{ Maximum } \\
\hline & & & & Lower Bound & Upper Bound & & \\
\hline Normal salivary gland & $16.72 \mathrm{e}$ & 1.65 & .74 & 14.67 & 18.77 & 14.25 & 17.90 \\
\hline Pleomorphic adenoma & $38.34 \mathrm{c}, \mathrm{d}$ & 8.44 & 3.77 & 27.86 & 48.82 & 27.09 & 50.29 \\
\hline $\begin{array}{c}\text { Papillary cyst adenoma } \\
\text { lymphomatosum }\end{array}$ & $29.68 \mathrm{~d}, \mathrm{e}$ & 5.70 & 2.55 & 22.60 & 36.76 & 23.12 & 38.38 \\
\hline Cribriform ACC & $41.83 \mathrm{c}, \mathrm{d}$ & 7.11 & 3.18 & 33.00 & 50.66 & 31.75 & 51.66 \\
\hline Tubular ACC & $47.27 \mathrm{c}$ & 8.44 & 3.78 & 36.78 & 57.75 & 38.96 & 58.58 \\
\hline Solid ACC & $64.61 \mathrm{a}, \mathrm{b}$ & 11.34 & 5.07 & 50.53 & 78.68 & 44.78 & 72.10 \\
\hline MEC (low grade) & $51.78 \mathrm{~b}, \mathrm{c}$ & 10.08 & 4.51 & 39.27 & 64.30 & 40.07 & 65.60 \\
\hline MEC (High grade) & $71.75 \mathrm{a}$ & 6.69 & 2.99 & 63.44 & 80.05 & 62.08 & 80.48 \\
\hline F value & \multicolumn{7}{|c|}{25.42} \\
\hline P value & \multicolumn{7}{|c|}{$<0.0001 *$} \\
\hline
\end{tabular}

Importance level $p<0.05$, *significant. Tukey's post hoc assessment: means having the same written above letter are not considerably different

\section{DISCUSSION}

In the current study, the distributions of an immune reaction of fascin were variable among different salivary gland neoplasms. Fascin expression showed the statistically significant higher value of area percent in malignant tumors (MEC and ACC) (55.45) than benign tumors (PA and Warthin's tumors (34.01); meanwhile, the normal salivary gland tissue showed the statistically significant least value (16.72). These results were in accordance with the study which found that the immunohistochemical appearance of fascin in malignant types of salivary gland tumor was meaningfully greater than that in normal salivary gland tissue ${ }^{(17)}$.

Regarding normal salivary gland tissues of the present study, fascin immunostaining was detected in the myoepithelium, cells of ducts and few acinus, while most of the acini were negatively stained. In breast tissue, fascin appearance was detected in myoepithelium in addition cells of lumen of few 
ducts and acini, where the presence of fascin immunostaining in myoepithelial cells augmented presence of actin filaments and cell motility that contribute to the beginning of cell motion and cell passage ${ }^{(18)}$. While no fascin reactivity was shown in follicular cells and $\mathrm{C}$ cells of the normal thyroid gland ${ }^{(19)}$. Another study revealed that fascin countenance has been found to be low or absent in normal epithelium of adult of variable origin. Nevertheless the height expression of fascin was in the basal layer of epithelium of nasopharynx, that augment the frequent observation and increased fascin expression in healthy, even though tumor neighboring epithelial tissue. The detected up regulated fascin appearance may reveal a tissue-exact appearance pattern or a link between fascin in addition to the multiplying capability of cells. Another explanation may be that the epithelial tissue that was surveyed was beside the tumor ${ }^{(20,21)}$.

Regarding benign tumors, both pleomorphic adenoma and Warthin's tumor showed higher fascin area percent than normal salivary gland tissue. These results were in agreement with another study that found that higher fascin expression in case of PA than normal salivary gland tissues ${ }^{(22)}$. Fascin immunostaining in PA was located in the nucleus and cytoplasm of epithelial, myoepithelial cells as well as in myxoid and chondroid tissue cells. This may be due to the role of fascin in the formation of cellular dendrite and pseudopodia that develop beyond the tumor's capsule and help the recurrence of the tumor. The increased fascin expression at the tumor boundaries indicates that fascin take part in the regulation of cell adhesion, in addition migration. Fascin has a part in the creation of the bundles in foremost edge projections of mesenchymal cells. These extensions of the cell membrane able to initiate intercellular contact ${ }^{(23)}$. While in Warthin's tumor, fascin immunostaining was located in the nucleus and cytoplasm mainly of basal cells of the ductal epithelium while the luminal cells showed faint cytoplasmic staining. Warthin's tumor is epithelial tumor where the character of staining of the basal and luminal epithelia varied, epithelial cells on luminal side characteristic immunologically similar to striated duct cells while those of the basal side close to the excretory duct basal cells. As basal cells of the duct have a accompaniment of actin filaments comparable to myoepithelial cells of acini and intercalated ducts ${ }^{(24)}$. So, this may clarify that the basal cells express more fascin immunostaining. Also, fascin reactivity is present in lymphocytes of the stroma of warthin's tumor, this is in accordance with another study that found that fascin was found to be expressed in the normal lymphoid tissue ${ }^{(25)}$.

In the current study, facin was located either in the cytoplasm or nucleus of the tumor cells. Fascin is present in the cytoplasm as inactive and active forms, the active one is conscripted to sites where filaments are in parallism, corresponding to the tips of $\Lambda$-precursors ${ }^{(26)}$. The active fascin concentration in the cytoplasm regulates the incidence of translocation of $\Lambda$-precursors to filopodia. When bound, fascin rises filopodial bundles stiffness and endorses the filopodia extension beyond the leading edge. Fast dynamics of fascin, while bound to actin filaments, is important for extension and maintenance of filopodia. Meanwhile, the nuclear localization of fascin is responsible for invasion regulation and is mediated via modifying several metastasis-associated genes ${ }^{(27)}$.

As regarding malignant tumors in the current study, the fascin expression was significantly higher in high-grade MEC (71.75) than low grade (51.78), this is in agreement with a study which found that fascin expression was correlated with high-grade type of OSCC than well-differentiated type indicating the fascin role in motility and migration of malignant cells ${ }^{(28)}$. Fascin was located in epidermoid cells cytoplasm and nucleus while mucous secreting cells were negatively stained. This may be explained by who found that podoplanin, (one of invasive markers similar to fascin in remodeling actin cytoskeleton) ${ }^{(29)}$, Fascin was found to be negatively expressed in mucous cells of mucoepidermoid carcinoma denoting that the expression is 
linked to degree of differentiation so terminally differentiated cells like mucous cells do not express podoplanin indicating poor function of these markers in mucous cells. In adenocarcinoma of the lung, the fascin immunostaining is mostly concentrated in the cytoplasm of epidermoid cells, while the surface ciliated and mucous cells of the epithelium of bronchi besides the seromucinous glands of the wall of the bronchi did not show fascin immunostaining ${ }^{(30)}$. Regarding adenoid cystic carcinoma, fascin was seen in cytoplasm and nucleus of malignant cells where solid pattern showing higher expression (64.61) than tubular (47.27) and cribriform patterns (41.83). These results are in accordance with those who found that the increased expressions of fascin are correlated with the high-grade ACC ${ }^{(17)}$, as fascin overexpression is linked with improved proliferation, cell motility, high possible for invasion and metastasis. Fascin allow cancer cells to invade into the matrix ${ }^{(26,31)}$.

\section{CONCLUSION}

Our study confirmed that fascin participates in the development of tumors of salivary glands and positively interrelated to the greatest aggressive patterns of malignant neoplasms.

\section{REFERENCES}

1- Sardar MA, Ganvir SM, Hazarey VK. A demographic study of salivary gland tumors. SRM 2018; 9:67-3.

2- Rito M, Fonseca I. Salivary gland neoplasms: Does morphological diversity reflect tumor heterogeneity. Pathobiology 2018; 85:85-9

3- Laishram RS, Kumar KA, Pukhrambam GD, Laishram S, Debnath K. Pattern of salivary gland tumors in Manipur, India: A 10-year study. South Asian J Cancer2013; 2:250-3.

4- Guzzo M, Laura D. Locati , Franz, J. Prott, Gatta G, McGurk M, Licitra L. Major and minor salivary gland tumors. $2^{\text {nd }}$ edition. Elsevier 2010; 134-48.

5- Hashimoto Y, Kim DJ, Adams JC. The roles of fascins in health and disease. J Pathol2011; 224: 289-300.

6- Jayo A, Parsons M. Fascin: a key regulator of cytoskeletal dynamics. Int J Biochem Cell Biol 2010; 42: 1614-7.
7- Jawhari AU, Buda A, Jenkins M, Shehzad K, Sarraf C. Fascin, an actin-bundling protein, modulates colonic epithelial cell invasiveness and differentiation in vitro. Am J Pathol2003; 162: 69-80.

8- Ang Li, Morton J, Yafeng MA, Karim S, Zhou Y, Faller J. Fascin is regulated by slug, promotes progression of pancreatic cancer in mice, and is associated with patient outcomes. J Gastroenterol. 2014; 1465: 1386-9.

9- Jayo A, Malboubi M, Antoku S, Chang W, Ortiz-Zapata E, Groen C, Pfisterer K, Tootle T, Charras G, Gundersen GG. Fascin regulates nuclear movement and deformation in migrating cells. Dev Cell2016; 38:371-83.

10- Konstantinos P, Christoph B, Irene S, Kai F, Haralampos G, James K, Wolf M, Juergen B, fascin upregulation in primary head and neck squamous cell carcinoma is associated with lymphatic metastasis oncology letters 2014; 7: 2041-46.

11- Vignjevic D, Schoumacher M, Gavert N, Janssen KP, Jih $\mathrm{G}$, Fascin, a novel target of beta-catenin-TCF signaling, is expressed at the invasive front of human colon cancer. Cancer Res 2007; 67: 6844-53.

12- Lee TK, Poon RT, Man K, Guan XY, Ma S, Fascin overexpression is associated with aggressiveness of oral squamous cell carcinoma. Cancer Lett2007; 254: 308-315.

13- Al-Alwan M, Olabi S, Ghebeh H, Barhoush E, Tulbah A, Fascin is a key regulator of breast cancer invasion that acts via the modification of metastasis-associated molecules. PLOS ONE 2011; 6: e27339.

14- Karasavvidou F, Barbanis S, Pappa D, Moutzouris G, Tzortzis V, Melekos MD, Koukoulis G, Fascin determination in urothelial carcinomas of the urinary bladder: a marker of invasiveness. Arch Pathol Lab Med 2008; 132: 19121915 .

15- EL Naggar AK, Chan JKC, Grandis JR, Takata T, Slootweg PJ, WHO classification of tumors of the head and neck. 4th ed. Lyon: IARC Press; 2017.

16- Ramos-Vara JA, Miller MA. Technical aspects of immunohistochemistry. Vet Pathol2014; 51:42-87.

17- Rodrigues P, Sawazaki-Calone I, De Oliveira C, Carneiro $\mathrm{C}$, Macedo S. Fascin promotes migration and invasion and is a prognostic marker for oral squamous cell carcinoma. Oncotarget2017; 843: 74736-54.

18- Youssef N, Hakim S. Association of Fascin and matrix metalloproteinase-9 expression with poor prognostic parameters in breast carcinoma of Egyptian women. Diagn Pathol2014; 9: 136-9. 
19- Chen G, Zhang F. Expression of fascin in thyroid neoplasms: A novel diagnostic marker. J Cancer Res Clin Oncol2008; 258:947-51.

20- Hashimoto Y, Skacel M, Lavery IC, Mukherjee AL, Casey G, Adams JC, Prognostic significance of fascin expression in advanced colorectal cancer: an immunohistochemical study of colorectal adenomas and adenocarcinomas. BMC Cancer 2006;6: 241.

21- Hu W, McCrea PD, Deavers M, Kavanagh JJ, Kudelka AP, Verschraegen $\mathrm{CF}$, Increased expression of fascin, motility associated protein, in cell cultures derived from ovarian cancer and in borderline and carcinomatous ovarian tumors. Clin Exp Metastasis 2000;18: 838.

22- Ann J, Christop D, Gosepath B, Kirkpatrick J, Jmann C. Recurrence of pleomorphic adenoma of the parotid gland-predictive value of cadherin-11 and fascin. APMIS 2008; 13:1050-7.

23- Rahrotaban S, Azmoudeh F, Kiyani SM. Fascin expression in pleomorphic adenoma and mucoepidermoid carcinoma. Dent Res J 2014; 11 3:370-4.

24- Segami N, Fukuda M, Manabe T. Immunohistological study of the epithelial components of Warthin's tumor. Int J Oral Maxillofac Surg 1989; 183:133-7.

25- Zhang Fa, Tao Li, Shen Z, Zhuo Li, Xu Li, En-Min Li.
Fascin expression in human embryonic, fetal, and normal adult tissue. J Histochem Cytochem 2008; 562: 193-9.

26- Vignjevic D, Kojima S, Aratyn Y, Danciu O, Svitkina T, Borisy GG.Role of fascin in filopodial protrusion. J Cell Biol2006, 174: 863-75.

27- Groen CM, Jayo A, Parsons M, Tina L, Tootle. Prostaglandins regulate nuclear localization of Fascin and its function in nucleolar architecture. Mol Biol Cell 2015; 2610: 1901-17.

28- Chaudhari RB. Cellular and molecular aspects at invasive tumor front in oral squamous cell carcinoma (part-I). SRM J Res Dent Sci2016; 7:166-72.

29- Kaur H, Gupta S. An analysis of the expression of Bcl-2, Podoplanin and lymphangiogenesis in benign and malignant salivary gland tumors. J Clin Exp Pathol2013; 14:120-5.

30- Choi PJ, Yung DK, Son CH, Lee KE, Lee JI, Roh MS. Fascin immunoreactivity for preoperatively predicting lymph node metastases in peripheral adenocarcinoma of the lung $3 \mathrm{~cm}$ or less in diameter.Eur J Cardiothorac Surg 2006; 30: 538-42.

31- Hayashi Y, Osanai M, Lee GH. Fascin-1 expression correlates with repression of E-cadherin expression in hepatocellular carcinoma cells and augments their invasiveness in combination with matrix metalloproteinases. Cancer Sci 2011; 1026:1228-35. 